\title{
Existence results for infinite systems of the Hilfer fractional boundary value problems in Banach sequence spaces
}

\section{Yousef Gholami ${ }^{1 *}$ (D)}

\section{"Correspondence: \\ y_gholami@sut.ac.ir \\ 'Department of Applied \\ Mathematics, Sahand University of \\ Technology, P.O. Box: 51335-1996, \\ Tabriz, Iran}

\begin{abstract}
The main aim of this paper is to present some existence criteria for an infinite system of Hilfer fractional boundary value problems of the form

$$
\mathcal{D}_{a^{+}}^{\alpha, \beta} u_{i}=-F_{i}(t, u), \quad u_{i}(a)=u_{i}(b)=0, a<t<b, i=1,2, \ldots,
$$

in Banach sequence spaces of $c_{0}$ and $I_{p}, p \geq 1$ types. Our approach is based on the Darbo-type fixed point theorems acting on the condensing operators. The obtained existence results in each of the above sequence spaces are illustrated by presenting some numerical examples.
\end{abstract}

MSC: Primary 34A08; 45A05; 47H08; secondary 26A33; 34B12;47H10

Keywords: Infinite systems of fractional differential equations; Hilfer fractional derivatives; Measure on noncompactness; Condensing operators; Darbo-type fixed point theorem; Existence of solutions

\section{Introduction}

Nowadays it is a well-known fact that one may take the integration and differentiation of arbitrary order in the appropriate functional spaces. The governing theory of such operations is known as fractional calculus, and consequently differential equations reconstructed in this theory are called fractional differential equations. So, it is a natural expectation that we try to re-obtain the fractional-order approaches of the current results belonging to the ordinary differential calculus. Between various versions of the fractionalorder differentiation the Hilfer fractional derivatives have an interesting technical property, which makes these derivatives much more important than other fractional-order derivatives, that is, the Hilfer fractional derivatives unify the Riemann-Liouville and Caputo fractional derivatives. This property makes the Hilfer fractional derivatives a more powerful mathematical tool in studying real-life phenomena and their engineering refinements. In this way, we suggest some resources for fractional differential equations such as $[1,5,7,8,11-15,19-22,25,27-29,31,32,34,36-40]$ and the references cited therein, for more consultation on this topic.

(c) The Author(s) 2021. This article is licensed under a Creative Commons Attribution 4.0 International License, which permits use, sharing, adaptation, distribution and reproduction in any medium or format, as long as you give appropriate credit to the original author(s) and the source, provide a link to the Creative Commons licence, and indicate if changes were made. The images or other third party material in this article are included in the article's Creative Commons licence, unless indicated otherwise in a credit line to the material. If material is not included in the article's Creative Commons licence and your intended use is not permitted by statutory regulation or exceeds the permitted use, you will need to obtain permission directly from the copyright holder. To view a copy of this licence, visit http://creativecommons.org/licenses/by/4.0/. 
In this paper we deal with one of the above-mentioned approaches, that is, the solvability assessment of an infinite system of the boundary value problems equipped with the Hilfer fractional derivatives. This fractional-order system can be formulated as

$$
\mathcal{D}_{a^{+}}^{\alpha, \beta} u_{i}=-F_{i}(t, u), \quad u_{i}(a)=u_{i}(b)=0, \quad a<t<b, i=1,2, \ldots,
$$

where

$\left(A_{1}\right) \mathcal{D}_{a^{+}}^{\alpha, \beta}$ denotes the Hilfer fractional derivative of order $1<\alpha \leq 2$ and type $0 \leq \beta \leq 1$.

$\left(A_{2}\right) \quad F_{i}(t, u):=a_{i}(t)+g_{i}\left(t, u_{1}, u_{2}, \ldots\right), i=1,2, \ldots$

$\left(A_{3}\right) \quad a_{i} \in C([a, b], \mathbb{R}), i=1,2, \ldots$

$\left(A_{4}\right) g_{i} \in C\left([a, b] \times \mathbb{R}^{\infty}\right), i=1,2, \ldots$.

As special cases of the infinite systems of the Hilfer fractional differential equations (1.1), one may consider the following instances:

1. The Hilfer fractional relaxation:

$$
F_{i}(t, u)=C u, \quad u:=\left(u_{i}\right)_{i=1}^{\infty}, C \in \mathbb{R} .
$$

2. The Hilfer fractional diffusion:

$$
F_{i}(t, u)=C \Delta u, \quad u:=\left(u_{i}\right)_{i=1}^{\infty}, C \in \mathbb{R},
$$

with the Laplacian $\Delta$.

The importance of the infinite systems of differential equations arises naturally in solving of partial differential equations. For instance, in the field of statistical physics infinite systems of ordinary differential equations appear as moment problems associated with the Fokker-Planck partial differential equations as described in [30]. As another application for the infinite systems of ordinary differential equations, one can consider the secondorder nonlinear partial differential equation of the form

$$
\frac{\partial^{2} u}{\partial t^{2}}-\frac{\partial^{2} u}{\partial y^{2}}=F\left(\frac{\partial u}{\partial y}, \frac{\partial u}{\partial t}, u, y, t\right)
$$

such that solving it can be reduced to solving the infinite system of the ordinary differential equations

$$
\frac{d^{2} x_{n}}{d t^{2}}+n^{2} x_{n}=f_{n}\left(t, x_{1}, \frac{d x_{1}}{d t}, x_{2}, \frac{d x_{2}}{d t}, \ldots\right), \quad n=1,2, \ldots
$$

More details can be found in [18] and the cited bibliography. Also, by the use of the longitudinal method of lines acting on the infinite systems of ordinary differential equations corresponding to some parabolic partial differential equations, one may obtain the approximate solutions of the partial differential equations as has been presented in detail in [35]. Besides, studying the infinite systems of differential equations leads to some significant problems in engineering, mechanics, theory of the branching processes, theory of the neural networks, and the theory of the dissociation of polymers (cf. [18, 23] and [24]). On the other hand, studying the infinite systems of differential equations on appropriate Banach spaces by themselves may occupy a distinguished place from the point 
of view of their solvability processes. As a consequence, one can consider the measures of noncompactness and constructed fixed point theory based on them as a pioneering technique to investigate the existence of solutions for the infinite systems of differential equations. Actually, measures of noncompactness from the point of view of applicability have a wide range of applications in providing solvability criteria for nonlinear differential equations via various refinements of the Darbo fixed point theorem. The history of measures of noncompactness originated with the Polish mathematician Kuratowski [17], and the first measure of noncompactness introduced by him was as follows:

$$
\alpha(S):=\inf \left\{\delta>0 \mid S=\bigcup_{i=1}^{n} S_{i} \text { for some } S_{i} \text { with } \operatorname{diam}\left(S_{i}\right) \leq \delta, 1 \leq i \leq n<\infty\right\},
$$

for the bounded subset $S$ of the metric space $X$. Besides, $\operatorname{diam}(T)$ denotes the diameter of the set $T \subset X$, i.e., $\operatorname{diam}(T):=\sup \{d(x, y) \mid x, y \in T\}$. However, from the point of view of applicability there is an another measure of noncompactness, which can be considered as the one most convenient and useful in applications. This measure is called the Hausdorff measure of noncompactness and is defined by

$$
\chi(S):=\inf \{\epsilon \mid S \text { has a finite } \epsilon \text {-net in } X\} .
$$

As a useful and compact collection of resources for more detailed consultation we refer to $[4,6,9]$ and $[10]$ and the references therein. From now on, for the sake of convenience we will use the abbreviation $\mathrm{MNC}$ for the measure of noncompactness.

Having the measures of noncompactness and related fixed point theory as our main technical tool, we now mention the following papers that are the inspiring resources of this paper, indeed. The authors in [23], studied the solvability of the infinite system of second-order periodic boundary value problem

$$
u_{i}^{\prime \prime}=-f_{i}\left(t, u_{1}, u_{2}, \ldots\right), \quad u_{i}(0)=u_{i}(T)=0, t \in[0, T], i=1,2, \ldots
$$

by the use of the measures of noncompactness and Darbo-type fixed point theorems on condensing operators. Motivated by this problem, and essentially following the applied technique in [23], we initiate studying the solvability of the infinite system of the Hilfer fractional boundary value problem (1.1).

At the end of this section, we state the organization of this investigation as follows. In Sect. 2, the Hilfer fractional derivatives and their importance in fractional calculus will be summarized. Besides, the concept of MNC and related Darbo-type fixed point theory will be presented. Section 3, is devoted to the some solvability criteria for the Hilfer fractional infinite system (1.1). We divide this section into the two parts. First, we define the Banach sequence space $c_{0}$, and consequently we provide some conditions on nonlinearities to obtain at least one solution for the infinite system (1.1). In the second part, presenting the Banach sequence space $l_{p}, p \geq 1$, we give some another sufficient conditions that lead us to the existence of at least one solution of the infinite system (1.1). Applicability of the obtained theoretical results in Sect. 3 will be illustrated in Sect. 4 by presenting some numerical examples for each existence criterion in $c_{0}$ and $l_{p}$. 


\section{Technical background}

In this section we first define the Hilfer fractional derivatives and their essential properties. So, we have the following definition.

Definition 2.1 ([15,33]) Assume $n-1<\alpha \leq n, n \in \mathbb{N}, 0 \leq \beta \leq 1$ and $f \in A C^{n}[a, b]$. Then the left and right sided Hilfer fractional derivatives of order $\alpha$ and type $\beta$, are defined as follows:

$$
\mathcal{D}_{a^{+}\left(b_{-}\right)}^{\alpha, \beta} f(t)= \begin{cases}\mathcal{D}_{a^{+}}^{\alpha, \beta} f(t):=\left(\mathcal{I}_{a^{+}}^{\beta(n-\alpha)} \mathcal{D}_{a^{+}}^{\alpha+\beta(n-\alpha)} f\right)(t), & t>a, \\ \mathcal{D}_{b_{-}}^{\alpha, \beta} f(t):=\left(\mathcal{I}_{b_{-}}^{\beta(n-\alpha)} \mathcal{D}_{b_{-}}^{\alpha+\beta(n-\alpha)} f\right)(t), & t<b .\end{cases}
$$

We note that in this definition, $\mathcal{I}_{a^{+}\left(b_{-}\right)}^{\alpha}$ stands for the left (right) sided Riemann-Liouville fractional integral of order $\alpha$ that acts on $f \in L(a, b)$ as follows [16]:

$$
\mathcal{I}_{a^{+}\left(b_{-}\right)}^{\alpha} f(t)= \begin{cases}\mathcal{I}_{a^{+}}^{\alpha} f(t)=\frac{1}{\Gamma(\alpha)} \int_{a}^{t}(t-s)^{\alpha-1} f(s) d s ; & \alpha>0, \\ \mathcal{I}_{b_{-}}^{\alpha} f(t)=\frac{1}{\Gamma(\alpha)} \int_{t}^{b}(s-t)^{\alpha-1} f(s) d s ; & \alpha>0, \\ f(t) ; & \alpha=0 .\end{cases}
$$

In addition, $\mathcal{D}_{a^{+}\left(b_{-}\right)}^{\alpha}$ stands for the Riemann-Liouville fractional derivative of order $\alpha$ that acts on $f \in A C^{n}[a, b]$ as follows [16]:

$$
\begin{aligned}
& \mathcal{D}_{a^{+}\left(b_{-} f\right.}^{\alpha} f(t) \\
& \quad= \begin{cases}\mathcal{D}_{a^{+}}^{\alpha} f(t):=\left(\frac{d^{n}}{d t^{n}}\right) \mathcal{I}_{a^{+}}^{n-\alpha} f(t)=\frac{1}{\Gamma(n-\alpha)}\left(\frac{d^{n}}{d t^{n}}\right) \int_{a}^{t}(t-s)^{n-\alpha-1} f(s) d s ; & \alpha>0, \\
\mathcal{D}_{b}^{\alpha} f(t):=(-1)^{n}\left(\frac{d^{n}}{d t^{n}}\right) \mathcal{I}_{b_{-}}^{n-\alpha} f(t)=\frac{(-1)^{n}}{\Gamma(n-\alpha)}\left(\frac{d^{n}}{d t^{n}}\right) \int_{t}^{b}(s-t)^{n-\alpha-1} f(s) d s ; & \alpha>0, \\
f(t) ; & \alpha=0,\end{cases}
\end{aligned}
$$

where $n=[\alpha]+1$.

Remark 2.2 Concentrating on the Hilfer fractional derivative $\mathcal{D}_{a^{+}\left(b_{-}\right)}^{\alpha, \beta}$, it follows that if we take $\beta:=0$, then this fractional derivative reduces to the Riemann-Liouville fractional derivatives, while taking $\beta:=1$, one obtains the left (right) sided Caputo fractional derivatives

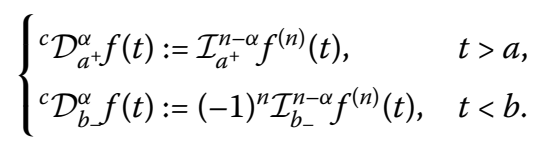

In the following lemma, the inversion formula for the Hilfer fractional derivatives is given.

Lemma 2.3 ([33]) Assume $n-1<\alpha \leq n, n \in \mathbb{N}$ and $0 \leq \beta \leq 1$. If $f \in L(a, b)$ and $\mathcal{I}_{a^{+}}^{(n-\alpha)(1-\beta)} f \in A C^{n-1}[a, b]$, then

$$
\left(\mathcal{I}_{a^{+}}^{\alpha} \mathcal{D}_{a^{+}}^{\alpha, \beta} f\right)(t)=f(t)-\sum_{k=0}^{n-1} \frac{(t-a)^{k-(n-\alpha)(1-\beta)}}{\Gamma(k-(n-\alpha)(1-\beta)+1)} \lim _{t \rightarrow a^{+}} \frac{d^{k}}{d x^{k}}\left(\mathcal{I}_{a^{+}}^{(n-\alpha)(1-\beta)} f\right)(t) .
$$


Table 1 Advantage of using the Hilfer fractional derivatives

\begin{tabular}{|c|c|}
\hline$\alpha, \beta$ & Corresponding reduced BVPs \\
\hline$\beta:=1$ & $\overline{\left(\mathcal{D}_{a^{+}}^{\alpha, \beta} u\right)(t)=-h(t), u(a)=u(b)=0 \rightarrow{ }^{c} \mathcal{D}_{a^{+}}^{\alpha} u(t)=-h(t), u(a)=u(b)=0}$ \\
\hline$\beta:=0$ & $\left(\mathcal{D}_{a^{+}}^{\alpha, \beta} u\right)(t)=-h(t), u(a)=u(b)=0 \longrightarrow \mathcal{D}_{a^{+}}^{\alpha} u(t)=-h(t), u(a)=u(b)=0$ \\
\hline$\alpha:=2$ & $\left(\mathcal{D}_{a^{+}}^{\alpha, \beta} u\right)(t)=-h(t), u(a)=u(b)=0 \longrightarrow u^{\prime \prime}(t)=-h(t), u(a)=u(b)=0$ \\
\hline
\end{tabular}

Now, we can represent the Green function corresponding to the infinite system of the Hilfer fractional boundary value problems (1.1).

Lemma 2.4 ([26]) Assume $h \in L(a, b)$. Then the Hilfer boundary value problem

$$
\left(\mathcal{D}_{a^{+}}^{\alpha, \beta} u\right)(t)=-h(t), \quad u(a)=u(b)=0, a<t<b,
$$

is equivalent to the integral equation

$$
u(t):=\int_{a}^{b} \mathcal{G}(t, s) h(s) d s
$$

where

$$
\mathcal{G}(t, s):=\frac{1}{\Gamma(\alpha)} \begin{cases}\left(\frac{t-a}{b-a}\right)^{1-(2-\alpha)(1-\beta)}(b-s)^{\alpha-1}-(t-s)^{\alpha-1} ; & a \leq s \leq t \leq b \\ \left(\frac{t-a}{b-a}\right)^{1-(2-\alpha)(1-\beta)}(b-s)^{\alpha-1} ; & a \leq t \leq s \leq b\end{cases}
$$

Lemma $2.5([26])$ For all $(t, s) \in[a, b] \times[a, b]$,

$$
\max |\mathcal{G}(t, s)|=\Lambda:=\frac{(b-a)^{\alpha-1}[\alpha-1+\beta(2-\alpha)]^{\alpha-1+\beta(2-\alpha)}(\alpha-1)^{\alpha-1}}{\Gamma(\alpha)\left[2 \alpha-2+\beta(2-\alpha]^{2 \alpha-2+\beta(2-\alpha)}\right.} .
$$

In this position, we are going to show the importance of the Hilfer fractional differential equations as a consequence of Remark 2.2.

Remark 2.6 Let us consider the Hilfer fractional boundary value problem (2.3). Table 1 illustrates that in view point of comprehensiveness, the Hilfer fractional differential equations include not only their related integer-order differential equations but also the Riemann-Liouville and Caputo versions of the corresponding fractional differential equations.

Consequently, the following table shows that the corresponding Green functions depend on the special values of the parameters $\alpha$ and $\beta$ and their maximums.

The first and second rows in Table 2 generalize the third row and the Hilfer-Green function (2.4) and its maximum, (2.5), generalizes the first and second rows. So, we arrive at the conclusion that on the most elementary level, the infinite system of Hilfer fractional boundary value problems (1.1) generalizes the research work [23], which was mentioned in the previous section.

It is time to have a quick overview on MNCs and the related fixed point theory. To this aim, we first state the following notations. Let $(E,\|\cdot\|)$ be a Banach space, $\mathbb{R}_{+}:=[0, \infty)$. If $X$ is a subset of $E$, then the symbols $\bar{X}$ and $\operatorname{Conv} X$ denote the closure and the convex 
Table 2 Corresponding Green functions and their maximums

\begin{tabular}{lll}
\hline$\alpha, \beta$ & $\mathcal{G}(t, s)$ & $\max \mathcal{G}(t, s)$ \\
\hline$\beta:=1$ & $\frac{1}{\Gamma(\alpha)} \begin{cases}\left(\frac{t-a}{b-a}\right)(b-s)^{\alpha-1}-(t-s)^{\alpha-1} ; & a \leq s \leq t \leq b, \\
\left(\frac{t-a}{b-a}\right)(b-s)^{\alpha-1} ; & a \leq t \leq s \leq b .\end{cases}$ \\
$\beta:=0$ & $\frac{1}{\Gamma(\alpha)} \begin{cases}\left(\frac{t-a}{b-a}\right)^{\alpha-1}(b-s)^{\alpha-1}-(t-s)^{\alpha-1} ; & a \leq s \leq t \leq b, \\
\left(\frac{t-a}{b-a}\right)^{\alpha-1}(b-s)^{\alpha-1} ; & a \leq t \leq s \leq b .\end{cases}$ \\
$\alpha:=2$ & $\begin{cases}\left(\frac{s-a}{b-a}\right)(b-t) ; & a \leq s \leq t \leq b, \\
\left(\frac{t-a}{b-a}\right)(b-s) ; & a \leq t \leq s \leq b .\end{cases}$ & $\frac{(b-a)^{\alpha-1}}{\Gamma(\alpha) \alpha^{\alpha}}$ \\
\hline
\end{tabular}

closure of $X$, respectively. Let $\mathfrak{M}_{E}$ and $\mathfrak{N}_{E}$ denote the families of the nonempty bounded and nonempty relatively compact subsets of $E$, respectively. We are now ready to define the concept of MNC.

Definition 2.7 ([2, 23]) A mapping $\mu: \mathfrak{M}_{E} \rightarrow \mathbb{R}_{+}$is said to be the measure of noncompactness in $E$ if it satisfies the following conditions:

$\left(A_{1}\right)$ The family $\operatorname{ker} \mu:=\left\{X \in \mathfrak{M}_{E}: \mu(X)=0\right\}$ is nonempty and $\operatorname{ker} \mu \subset \mathfrak{N}_{E}$.

$\left(A_{2}\right) X \subset Y \Longrightarrow \mu(X) \leq \mu(Y)$.

(A3) $\mu(\bar{X})=\mu(X)$.

$\left(A_{4}\right) \mu(\operatorname{Conv} X)=\mu(X)$.

$\left(A_{5}\right) \mu(\lambda X+(1-\lambda) Y) \leq \lambda \mu(X)+(1-\lambda) \mu(Y)$ for $\lambda \in[0,1]$.

$\left(A_{6}\right)$ If $\left\{X_{n}\right\}$ is a sequence of closed sets in $\mathfrak{M}_{E}$, such that $X_{n+1} \subset X_{n}, n=1,2, \ldots$ and if $\lim _{n \rightarrow \infty} \mu\left(X_{n}\right)=0$, then $X_{\infty}:=\bigcap_{n=1}^{\infty} X_{n} \neq \emptyset$.

If an MNC satisfies the following additional conditions, we call it a regular MNC:

$\left(A_{7}\right) \mu(X \cup Y)=\max \{\mu(X), \mu(Y)\}$.

$\left(A_{8}\right) \mu(X+Y) \leq \mu(X)+\mu(Y)$.

$\left(A_{9}\right) \mu(\lambda X)=|\lambda| \mu(X)$ for $\lambda \in \mathbb{R}$.

$\left(A_{10}\right) \operatorname{ker} \mu=\mathfrak{N}_{E}$.

Since our solvability approach will be constructed by the Darbo-type fixed point theorem on the condensing operators, we first have to present the classic Darbo fixed point theorem and definition of a condensing operator.

Theorem 2.8 (Darbo fixed point theorem, [3, 23]) Let $\Omega$ be a nonempty, bounded, closed and convex subset of a Banach space $E$ and let $T: \Omega \rightarrow \Omega$ be a continuous mapping such that there exists a constant $k \in[0,1)$ with the property

$$
\mu(T(\Omega)) \leq k \mu(\Omega)
$$

Then $T$ has a fixed point in the set $\Omega$.

Let us mention the fact that, in the Darbo fixed point theorem, $\mu$ stands for the Kuratowski MNC. In what follows we are going to present a generalization of the Darbo fixed point theorem that admits every arbitrary MNC to obtain at least one fixed point. To this aim, we define the concept of condensing operators.

Definition 2.9 ([3,23]) Let $E_{i}, i=1,2$ be two Banach spaces and $\mu_{i}, i=1,2$ be two arbitrary MNCs on $E_{i}, i=1,2$, respectively. An operator $T: E_{1} \rightarrow E_{2}$ is called a $\left(\mu_{1}, \mu_{2}\right)$-condensing 
operator if it is continuous and $\mu_{2}(T(\Omega))<\mu_{1}(\Omega)$, for every bounded noncompact set $\Omega \subset E_{1}$.

We note that if $E_{1}=E_{2}$ and $\mu_{1}=\mu_{2}$, then $T$ is called a $\mu$-condensing operator.

As stated above we are interested in the solvability processes represented in [23]. Thus, we shall consider a particular class of the condensing operators, the so-called the Meirkeeler condensing operators that are defined as follows.

Definition 2.10 ([3,23]) Let $C$ be a nonempty subset of a Banach space $E$ and let $\mu$ be an arbitrary MNC on $E$. We say that an operator $T: C \rightarrow C$ is a Meir-Keeler condensing operator if for any $\epsilon>0$, there exists $\delta>0$ such that

$$
\epsilon \leq \mu(X)<\epsilon+\delta \quad \Longrightarrow \quad \mu(T(X))<\epsilon,
$$

for any bounded subset $X$ of $C$.

Theorem 2.11 ([3,23]) Let $C$ be a nonempty, bounded, closed and convex subset of a Banach space $E$ and let $\mu$ be an arbitrary MNC on E. If $T: C \rightarrow C$ is a continuous and condensing operator, then $T$ has a fixed point and the set of all of fixed points of $T$ in $C$ is compact.

We finalize this section with formulating the Hausdorff MNC in two Banach sequence spaces $c_{0}$ and $l_{1}$. More details can be found in ([10], pp. 162-163, Th. 5.18, (a), (b)).

In the Banach sequence space $\left(c_{0},\|\cdot\|_{c_{0}}\right)$, the Hausdorff MNC $\chi$ can be formulated as

$$
\chi(B):=\lim _{n \rightarrow \infty}\left\{\sup _{u \in B}\left[\max _{k \geq n}\left|u_{k}\right|\right]\right\}, \quad B \in \mathfrak{M}_{c_{0}}
$$

in which $u(t)=\left\{u_{i}(t)\right\}_{i=1}^{\infty} \in c_{0}$, for each $t \in[a, b]$.

Furthermore, in the Banach sequence space $l_{p}, p \geq 1$, the Hausdorff MNC $\chi$ can be formulated as

$$
\chi(B):=\lim _{n \rightarrow \infty}\left\{\sup _{u \in B}\left[\sum_{k \geq n}\left|u_{k}\right|^{p}\right]^{\frac{1}{p}}\right\}, \quad B \in \mathfrak{M}_{l_{p}},
$$

in which $u(t)=\left\{u_{i}(t)\right\}_{i=1}^{\infty} \in l_{1}$, for each $t \in[a, b]$.

\section{Existence criteria via measures of noncompactness in Banach sequence spaces}

As discussed above, depending on the Banach sequence spaces $c_{0}$ and $l_{p}$ we divide this section into two parts and then we attempt to present solvability criterion in each case for the infinite system of the Hilfer fractional boundary value problems (1.1). From now on by a MNC, we mean the Hausdorff measure of noncompactness $\chi$ defined by (2.8) and (2.9).

\subsection{Existence criterion in $c_{0}$}

To begin with, we have to consider the following hypotheses: 
C.1 $F_{i}:[a, b] \times \mathbb{R}^{\infty} \rightarrow \mathbb{R}$ and the operator $\mathcal{Q}:[a, b] \times c_{0} \rightarrow c_{0}$ defined as

$$
(\mathcal{Q} u)(t):=\left(F_{1}(t, u), F_{2}(t, u), F_{3}(t, u), \ldots\right)
$$

such that the class of all functions $((\mathcal{Q} u)(t))_{t \in[a, b]}$ is equicontinuous at every point of the sequence space $c_{0}$.

C.2 $a_{i}(t) \in C\left([a, b] ; \mathbb{R}_{+}\right), i=1,2, \ldots$ and the sequence $\left\{a_{i}\right\}_{i=1}^{\infty}$ converges uniformly on $[a, b]$ to a function identically vanishing on $[a, b]$.

C.3 $\left|g_{i}\left(t, u_{1}, u_{2}, u_{3}, \ldots\right)\right| \leq b_{i}(t)+q_{i}(t) \sup _{k \geq i}\left\{\left|u_{k}\right|\right\}, i=1,2, \ldots$ such that $\left\{b_{i}\right\}_{i=1}^{\infty}$ has the same conditions as asserted in (C.2) and $q_{i}(t) \in C\left([a, b] ; \mathbb{R}_{+}\right)$and the sequence $\left\{q_{i}\right\}_{i=1}^{\infty}$ is equibounded on $[a, b]$.

Before presenting the first main result, for the sake of convenience we set the following notation:

1. $\mathcal{A}:=\sup \left\{a_{i}(t)+b_{i}(t) \mid t \in[a, b], i \in \mathbb{N}\right\}$.

2. $Q:=\sup \left\{q_{i}(t) \mid t \in[a, b], i \in \mathbb{N}\right\}$.

Theorem 3.1 Assume that the hypotheses (C.1)-(C.3) are satisfied. Then the infinite system of the Hilfer fractional boundary value problems (1.1) has at least one solution $u(t)=\left(u_{i}(t)\right)_{i}$ provided that $\Lambda(b-a) Q<1$, in which for each $t \in[a, b], u(t) \in c_{0}$. Moreover, for each $t \in[a, b], u(t) \in \operatorname{ker} \chi$.

Proof At the beginning let us recall the conclusion of Lemma 2.4, that is the infinite system of the Hilfer fractional boundary value problems (1.1), is equivalent to the infinite system of the integral equations

$$
u_{i}(t):=\int_{a}^{b} \mathcal{G}(t, s) F_{i}(s, u(s)) d s, \quad i=1,2, \ldots
$$

Note that, since in the Green function $\mathcal{G}(t, s)$ defined by $(2.5)$, we have

$$
\mathcal{G}(a, s)=0, \quad \mathcal{G}(b, s)=0,
$$

if we define the fixed point problem

$$
\left(\mathcal{F}_{i} u\right)(t):=\int_{a}^{b} \mathcal{G}(t, s) F_{i}(s, u(s)) d s, \quad i=1,2, \ldots,
$$

it is clear that

$$
\left(\mathcal{F}_{i} u\right)(a)=0, \quad\left(\mathcal{F}_{i} u\right)(b)=0 .
$$

In other mean, the integral operators $\mathcal{F}_{i} u, i=1,2, \ldots$, satisfy the boundary conditions of (1.1).

Having this fixed point problem in hand, we now able to prove that all conditions of Theorem 2.11 hold. So, let us begin as follows.

According to the (3.1), we get

$$
\|u\|_{c_{0}}=\max _{i \geq 1}\left|\int_{a}^{b} \mathcal{G}(t, s) F_{i}(s, u(s)) d s\right|
$$




$$
\begin{aligned}
& \leq \max _{i \geq 1} \int_{a}^{b}\left|\mathcal{G}(t, s) F_{i}(s), u(s)\right| d s \\
& \leq \Lambda(b-a) \max _{i \geq 1}\left[\sup _{s \in[a, b]}\left(a_{i}(s)+b_{i}(s)+q_{i}(s)\left|u_{i}(s)\right|\right)\right] \\
& \leq \Lambda(b-a)\left[\mathcal{A}+Q\|u\|_{c_{0}}\right] .
\end{aligned}
$$

So, we have

$$
\|u\|_{c_{0}} \leq \frac{\Lambda(b-a) \mathcal{A}}{1-\Lambda(b-a) Q}:=\theta
$$

Here we start with introducing the nonempty, bounded, closed and convex subset of a Banach space $c_{0}$. Consider $u^{0}(t):=\left(u_{i}^{0}(t)\right), t \in[a, b]$ in which for each $i=1,2,3, \ldots, u_{i}^{0}(t):=$ 0 . In this case $\bar{B}\left(u^{0} ; r\right)$ denoting the closed ball centered at $u^{0}$, and having radius $r \leq \theta$, coincides with all conditions of the subset $C$ in Theorem 2.11. Now, it is time to define an operator $T$ on $\bar{B}$ satisfying all conditions mentioned in Theorem 2.11, that is, $T$ must be continuous and a Meir-Keeler condensing operator on $\bar{B}$. To this aim, we refer to the operators $\left(\mathcal{F}_{i} u\right)(t), i=1,2,3, \ldots$ defined by (3.2) and define the operator $T$ on $C([a, b], \bar{B})$ for each $t \in[a, b]$ as follows:

$$
(T u)(t):=\left\{\left(\mathcal{F}_{i} u\right)(t)\right\}=\left\{\int_{a}^{b} \mathcal{G}(t, s) F_{i}(s, u(s)) d s\right\}, \quad i=1,2, \ldots
$$

such that $u(t):=\left(u_{i}(t)\right) \in \bar{B}$ and $u_{i}(t) \in C([a, b], \mathbb{R})$ for $i=1,2,3, \ldots$

In accordance with the hypothesis (C.1), we prove that, for each $t \in[a, b],(T u)(t) \in c_{0}$. Indeed, since for each $t \in[a, b],\left(F_{i}(t, u(t))\right) \in c_{0}$, it follows that

$$
\begin{aligned}
\lim _{i \rightarrow \infty}(T u)(t) & :=\lim _{i \rightarrow \infty}\left\{\int_{a}^{b} \mathcal{G}(t, s) F_{i}(s, u(s)) d s\right\} \\
& =\left\{\int_{a}^{b} \mathcal{G}(t, s) \lim _{i \rightarrow \infty} F_{i}(s, u(s)) d s\right\} \\
& =0 .
\end{aligned}
$$

To proceed, we recall these basic facts; as a result of the hypothesis (C.1), the operator $T$ defined by (3.5) belongs to $C([a, b], \bar{B})$. Besides, according to (3.2) and (3.4), it is clear that

$$
\left\|(T u)-u^{0}\right\|_{c_{0}}=\|T u\|_{c_{0}} \leq \theta
$$

Thus, $T$ is self-mapping on $\bar{B}$. Furthermore, because of the (3.3), $T$ satisfies the boundary conditions of the infinite system of the Hilfer fractional boundary value problems (1.1) that is $(T u)(a)=0$ and $(T u)(b)=0$.

Looking back into the proof and compare it with the proof of Theorem 2.11, it just remains to prove that the operator $T$ is a Meir-Keeler condensing operator. To this aim, for each arbitrary $\epsilon>0$ we have to find an $\delta>0$ such that

$$
\epsilon \leq \chi(\bar{B})<\epsilon+\delta \quad \Longrightarrow \quad \chi(T(\bar{B}))<\epsilon
$$


Here we recall the $c_{0}$-definition of the Hausdorff MNC presented in (2.8), and apply it as follows:

$$
\begin{aligned}
\chi(T(\bar{B})) & :=\lim _{n \rightarrow \infty}\left(\sup _{u(t) \in \bar{B}}\left[\max _{i \geq n}\left|\int_{a}^{b} \mathcal{G}(t, s) F_{i}(s, u(s)) d s\right|\right]\right) \\
& \leq \lim _{n \rightarrow \infty}\left(\sup _{u(t) \in \bar{B}}\left[\max _{i \geq n}\left|\int_{a}^{b} \mathcal{G}(t, s)\left\{a_{i}(s)+b_{i}(s)+q_{i}(s) \sup _{i \geq n}\left|u_{i}(s)\right|\right\} d s\right|\right]\right) \\
& \leq \lim _{n \rightarrow \infty}\left(\sup _{u(t) \in \bar{B}}\left[\max _{i \geq n}\left|Q \sup _{i \geq n}\right| u_{i}\left|\int_{a}^{b} \mathcal{G}(t, s) d s\right|\right]\right) \\
& <Q \Lambda(b-a) \lim _{n \rightarrow \infty}\left(\sup _{u \in \bar{B}}\left[\max _{i \geq n}\left|u_{i}\right|\right]\right) \\
& =\chi(\bar{B}) Q \Lambda(b-a) .
\end{aligned}
$$

Now, it is enough to choose

$$
\delta:=\frac{\epsilon(1-\Lambda(b-a) Q)}{\Lambda(b-a) Q}
$$

to obtain $\chi(T(\bar{B}))<\epsilon$. This means that the operator $T$ is a Meir-Keeler condensing operator on $\bar{B} \subset c_{0}$. So, $T$ has at least one fixed point in $\bar{B}$ that is a solution of the infinite system of the Hilfer fractional boundary value problems (1.1). In addition, if we consider the fixed point set

$$
\mathcal{K}:=\{u \in \bar{B}: u=T u\}
$$

clearly $\mathcal{K}$ is closed and nonempty $\left(u^{0} \in \mathcal{K}\right)$. Moreover, since $\mathcal{K} \subset \operatorname{Conv}\left(T \mathcal{K} \cup\left\{u^{0}\right\}\right)$, it follows that

$$
\chi(\mathcal{K}) \leq \chi\left(T \mathcal{K} \cup\left\{u^{0}\right\}\right)=\chi(T \mathcal{K}) \leq k \chi(\mathcal{K}), \quad k \in(0,1) .
$$

So, we arrive at the conclusion that $\mathcal{K} \subset \operatorname{ker} \chi$. This completes the proof.

\subsection{Existence criterion in $I_{p}$}

Guaranteeing the existence at least one solution for the infinite system of the Hilfer fractional boundary value problems (1.1) in Banach sequence space $l_{p}$ requires the following general assumptions:

L.1 $F_{i}:[a, b] \times \mathbb{R}^{\infty} \rightarrow \mathbb{R}$ and the operator $\mathcal{S}:[a, b] \times l_{p} \rightarrow l_{p}$ defined as

$$
(\mathcal{S} u)(t):=\left(F_{1}(t, u), F_{2}(t, u), F_{3}(t, u), \ldots\right)
$$

such that the class of all functions $((\mathcal{S} u)(t))_{t \in[a, b]}$ is equicontinuous at every point of the sequence space $l_{p}$.

L.2 $a_{i}(t) \in C\left([a, b] ; \mathbb{R}_{+}\right), i=1,2, \ldots$, and the function series $\sum_{i=1}^{\infty} a_{i}(t)$ is uniformly convergent. 
L.3 For any natural $i$, there exists a function $q_{i}(t) \in C\left([a, b] ; \mathbb{R}_{+}\right)$such that

$$
\left|g_{i}\left(t, u_{1}, u_{2}, u_{3}, \ldots\right)\right|^{p} \leq b_{i}(t)+q_{i}(t)\left|u_{i}\right|^{p}
$$

holds for $t \in[a, b]$ and $u=\left(u_{i}\right) \in l_{p}$ for $i=1,2, \ldots$, the sequence $\left\{b_{i}(t)\right\}_{i=1}^{\infty}$ behaves as mentioned in the hypothesis (L.2) and the sequence $\left\{q_{i}(t)\right\}_{i=1}^{\infty}$ is equibounded on $[a, b]$.

Similar to the previous section, we introduce here the following finite constants:

1. $\mathcal{A}:=\sup \{g(t) \mid t \in[a, b]\}$, such that $g(t):=\sum_{i=1}^{\infty}\left(a_{i}(t)+b_{i}(t)\right)$ is a continuous and uniformly convergent series function on $[a, b]$.

2. $Q:=\sup \{q(t) \mid t \in[a, b]\}$, in which $q(t):=\sum_{i=1}^{\infty} q_{i}(t)$ is a continuous series function on $[a, b]$.

Here we are ready to state and prove the second existence criterion for the infinite system of the Hilfer fractional boundary value problems (1.1). So, we have the following theorem.

Theorem 3.2 Assume that the hypotheses (L.1)-(L.3) hold. Then the infinite system of the integral equations (3.1) has at least one solution $u(t)=\left(u_{i}(t)\right)_{i}$, provided that $\Lambda^{p}(b-a)^{\frac{p+q}{q}} Q<1, \frac{1}{p}+\frac{1}{q}=1$, in which for each $t \in[a, b], u(t) \in l_{p}$. Moreover, for each $t \in[a, b], u(t) \in \operatorname{ker} \chi$.

Proof Since in Sects. 3.1 and 3.2 the infinite system (1.1) must be studied, in the proof of this theorem we try to skip those parts that are the same as those in Theorem 3.1. So, we do not repeat the first part of the proof of Theorem, (3.1) up to (3.3), here. Therefore, we start as follows. Consider the space $C\left([a, b] ; l_{p}\right)$ of all continuous functions on $[a, b]$ with values in the space $l_{p}$ and endowed with the classic norm

$$
\|u\|:=\sup \left\{\|u(t)\|_{l_{p}}: t \in[a, b]\right\}
$$

We now define the operator $\mathcal{L}$ on the space $C\left([a, b] ; l_{p}\right)$ as

$$
(\mathcal{L} u)(t):=\left\{\left(\mathcal{F}_{i} u\right)(t)\right\}:=\left\{\int_{a}^{b} \mathcal{G}(t, s) F_{i}(s, u(s)) d s\right\}
$$

Accordingly, for a fixed $u(t)=\left(u_{i}(t)\right) \in C\left([a, b] ; l_{p}\right)$, for each $t \in[a, b]$ and by the use of the Hölder inequality, we have

$$
\begin{aligned}
\|\mathcal{L} u(t)\|_{l_{p}}^{p} & =\sum_{i=1}^{\infty}\left|\int_{a}^{b} \mathcal{G}(t, s) F_{i}(s, u(s)) d s\right|^{p} \\
& \leq \sum_{i=1}^{\infty}\left(|\mathcal{G}(t, s)|^{p}\left|F_{i}(s, u(s))\right|^{p} d s\right)\left(\int_{a}^{b} d s\right)^{\frac{p}{q}} \\
& \leq(b-a)^{\frac{p}{q}} \sum_{i=1}^{\infty} \int_{a}^{b}|\mathcal{G}(t, s)|^{p}\left[a_{i}(s)+b_{i}(s)+q_{i}(s)\left|u_{i}(s)\right|^{p}\right] d s \\
& \leq(b-a)^{\frac{p}{q}} \sum_{i=1}^{\infty}\left[\int_{a}^{b}|\mathcal{G}(t, s)|^{p}\left(a_{i}(s)+b_{i}(s)\right) d s+\int_{a}^{b}|\mathcal{G}(t, s)|^{p} q_{i}(s)\left|u_{i}(s)\right|^{p} d s\right]
\end{aligned}
$$




$$
\begin{aligned}
& \leq \Lambda^{p}(b-a)^{\frac{p}{q}} \sum_{i=1}^{\infty}\left[\int_{a}^{b}\left(a_{i}(s)+b_{i}(s)\right) d s+\int_{a}^{b} q_{i}(s)\left|u_{i}(s)\right|^{p} d s\right] \\
& =\Lambda^{p}(b-a)^{\frac{p}{q}}\left[\int_{a}^{b} \sum_{i=1}^{\infty}\left(a_{i}(s)+b_{i}(s)\right) d s+\int_{a}^{b} \sum_{i=1}^{\infty} q_{i}(s)\left|u_{i}(s)\right|^{p} d s\right] \\
& \leq \Lambda^{p}(b-a)^{\frac{p}{q}}\left[\int_{a}^{b} g(s) d s+Q \int_{a}^{b} \sum_{i=1}^{\infty}\left|u_{i}(s)\right|^{p} d s\right] \\
& \leq \Lambda^{p}(b-a)^{\frac{p+q}{q}}\left[\mathcal{A}+Q\|u\|_{l_{p}}^{p}\right],
\end{aligned}
$$

where $q>1$ is the conjugate number of $p$, that is, $\frac{1}{p}+\frac{1}{q}=1$. So, we get the following:

$$
\|\mathcal{L} u\|_{l_{p}}^{p} \leq \Lambda^{p}(b-a)^{\frac{p+q}{q}}\left[\mathcal{A}+Q\|u\|_{l_{p}}^{p}\right]
$$

This inequality ensures that $\mathcal{L} u$ is bounded on the interval $[a, b]$ that guarantees that the operator $\mathcal{L}$ transforms the space $C\left([a, b] ; l_{p}\right)$ into itself. Besides, considering the fixed point problem (3.1)-(3.2), we obtain the following inequality:

$$
\|u\|_{l_{p}}^{p} \leq \Lambda^{p}(b-a)^{\frac{p+q}{q}}\left[\mathcal{A}+Q\|u\|_{l_{p}}^{p}\right]
$$

which leads us to the following straightforward inequality:

$$
\|u\| \leq \frac{\Lambda(b-a)^{\frac{p+q}{p q}} \mathcal{A}^{\frac{1}{p}}}{\left(1-\Lambda^{p}(b-a)^{\frac{p+q}{q}} Q\right)^{\frac{1}{p}}}:=\vartheta .
$$

In this position, we are ready to show that all conditions of Theorem 2.11 are satisfied. First, similar to Theorem 3.1, we consider the nonempty, bounded, closed and convex set $\bar{B}\left(u^{0}, r\right) \subset l_{p}$ as defined in Theorem 3.1 with $r \leq \vartheta$. Next, since based on the hypothesis (L.1) the operator $\mathcal{L}$ is continuous on $C([a, b] ; \bar{B})$, it is just enough to prove that for each $t \in$ $[a, b]$ the operator $\mathcal{L}$ defined by (3.6) is a Meir-Keeler condensing operator on $C([a, b] ; \bar{B})$. To this end, for an arbitrary $\epsilon>0$ we find an $\delta>0$ such that if $\epsilon \leq \chi(\bar{B})<\epsilon+\delta$, then $\chi(\mathcal{L}(\bar{B}))<\epsilon$. To achieve the desired result, we use the $l_{p}$-definition of the Hausdorff MNC $\chi$ represented by (2.9). Hence, in the light of the Hölder inequality we get

$$
\begin{aligned}
\chi(\mathcal{L}(\bar{B}))^{p} & =\leq\left\{\lim _{n \rightarrow \infty}\left(\sup _{u(t) \in \bar{B}}\left[\sum_{i \geq n} \int_{a}^{b} \mathcal{G}(t, s) F_{i}(s, u(s)) d s\right]\right)\right\}^{p} \\
& \leq \lim _{n \rightarrow \infty}\left(\sup _{u(t) \in \bar{B}}\left[\sum_{i \geq n}\left|\int_{a}^{b} \mathcal{G}(t, s) F_{i}(s, u(s)) d s\right|^{p}\right]\right) \\
& \leq \lim _{n \rightarrow \infty}\left(\sup _{u(t) \in \bar{B}}\left[\sum_{i \geq n} \int_{a}^{b}\left|\mathcal{G}(t, s) F_{i}(s, u(s))\right|^{p} d s\left(\int_{a}^{b} d s\right)^{\frac{p}{q}}\right]\right) \\
& \leq(b-a)^{\frac{p}{q}} \lim _{n \rightarrow \infty}\left(\sup _{u(t) \in \bar{B}}\left[\sum_{i \geq n} \int_{a}^{b}\left|\mathcal{G}(t, s)^{p}\right|\left[a_{i}(s)+b_{i}(s)+q_{i}(s)\left|u_{i}(s)\right|^{p}\right] d s\right]\right) \\
& \leq \Lambda^{p}(b-a)^{\frac{p}{q}} \lim _{n \rightarrow \infty}\left(\sup _{u(t) \in \bar{B}}\left[\sum_{i \geq n} \int_{a}^{b}\left[a_{i}(s)+b_{i}(s)+q_{i}(s)\left|u_{i}(s)\right|^{p}\right] d s\right]\right)
\end{aligned}
$$




$$
\leq \Lambda^{p}(b-a)^{\frac{p}{q}} Q \chi(\bar{B})^{p}
$$

where $q$ is the conjugate number of $p$. So, we have

$$
\chi(\mathcal{L}(\bar{B}))^{p} \leq \Lambda^{p}(b-a)^{\frac{p}{q}} Q \chi(\bar{B})^{p} .
$$

Equivalently, it follows that

$$
\chi(\mathcal{L}(\bar{B})) \leq \Lambda(b-a)^{\frac{p+q}{p q}} Q^{\frac{1}{p}} \chi(\bar{B}) .
$$

Therefore, choosing

$$
\delta:=\frac{\epsilon\left(1-\Lambda(b-a)^{\frac{p+q}{p q}} Q^{\frac{1}{p}}\right)}{\Lambda(b-a)^{\frac{p+q}{p q}} Q^{\frac{1}{p}}},
$$

we arrive at the conclusion that if $\epsilon \leq \chi(\bar{B})<\epsilon+\delta$, then $\chi(\mathcal{L}(\bar{B}))<\epsilon$. In other words, the operator $\mathcal{L}$ is a Meir-Keeler condensing operator on $C([a, b] ; \bar{B})$. So, according to Theorem 2.11, the infinite system of integral equations (3.1) has at least one solution in $\bar{B} \subset l_{p}$. Furthermore, the set of all such solutions belongs to $\operatorname{ker} \chi$ as showed in Theorem 3.1. This completes the proof.

\section{Numerical examples}

Example 4.1 We start this section with the following infinite system of the Hilfer fractional boundary value problems:

$$
\mathcal{D}_{a^{+}}^{\alpha, \beta} u_{i}=-a_{i}(t)-g_{i}\left(t, u_{1}, u_{2}, \ldots\right), \quad u_{i}(a)=u_{i}(b)=0, a<t<b, i=1,2, \ldots,
$$

subject to the following setting:

$$
\begin{aligned}
& a_{i}(t)=:=\frac{(b-t)(t-a)}{i^{2} E_{\alpha, B}(i t)} \\
& g_{i}\left(t, u_{1}, u_{2}, \ldots\right):=\sum_{k=i}^{\infty} \frac{E_{\alpha, \beta}(t)}{k^{2}(1+i)} \cdot \frac{u_{k}}{1+(k-i)^{4} u_{k}^{4}}, \quad i=1,2, \ldots
\end{aligned}
$$

where

$$
1<\alpha \leq 2, \quad \beta:=0.5, \quad B:=\alpha+0.5(2-\alpha), \quad a:=0, \quad b:=1 .
$$

Note that by $E_{\alpha, \beta}(t)$ we mean the two-parametric Mittag-Leffler function

$$
E_{\alpha, \beta}(t):=\sum_{k=0}^{\infty} \frac{t^{k}}{\Gamma(\alpha k+\beta)}, \quad \alpha>0, \beta \in \mathbb{C} .
$$

For the sake of better applicability, we choose $p:=2$, that is, we are migrating into the Banach sequence space $l_{2}$. Here, we begin by proving that all of the hypotheses (L.1)(L.3) are satisfied. But formerly we paid attention to the key point that, for an arbitrary 
positive constant $\rho$, it follows that

$$
\max _{\omega>0} \frac{\omega}{1+\rho^{4} \omega^{4}}:=\frac{3^{\frac{3}{4}}}{4 \rho} .
$$

We fix the arbitrary natural number $i$ to get the following:

$$
\begin{aligned}
& \left|F_{i}\left(t, u_{1}, u_{2}, \ldots\right)\right|^{2} \\
& \quad:=\left|a_{i}(t)-g_{i}\left(t, u_{1}, u_{2}, \ldots\right)\right|^{2} \\
& \quad=\left|\frac{(1-t) t}{i^{2} E_{\alpha, B}(i t)}+\sum_{k=i}^{\infty} \frac{E_{\alpha, \beta}(t)}{k^{2}(1+i)} \cdot \frac{u_{k}}{1+(k-i)^{4} u_{k}^{4}}\right|^{2} \\
& \quad \leq 2\left\{\left[\frac{(1-t) t}{i^{2} E_{\alpha, B}(i t)}\right]^{2}+\left[\sum_{k=i}^{\infty} \frac{E_{\alpha, \beta}(t)}{k^{2}(1+i)} \cdot \frac{u_{k}}{1+(k-i)^{4} u_{k}^{4}}\right]^{2}\right\} \\
& \quad \leq 2\left\{\left[\frac{(1-t) t}{i^{2} E_{\alpha, B}(i t)}\right]^{2}+\left[\left(\sum_{k=i}^{\infty}\left(\frac{E_{\alpha, \beta}(t)}{k^{2}(1+i)}\right)^{2}\right)^{\frac{1}{2}}\left(\sum_{k=i}^{\infty}\left(\frac{u_{k}}{1+(k-i)^{4} u_{k}^{4}}\right)^{2}\right)^{\frac{1}{2}}\right]^{2}\right\} \\
& \quad \leq 2\left[\frac{(1-t) t}{i^{2} E_{\alpha, B}(i t)}\right]^{2}+2 \sum_{k=i}^{\infty}\left(\frac{E_{\alpha, \beta}(t)}{k^{2}(1+i)}\right)^{2} \cdot \sum_{k=i}^{\infty}\left(\frac{u_{k}}{1+(k-i)^{4} u_{k}^{4}}\right)^{2} \cdot
\end{aligned}
$$

It is time to recall the max value (4.4), which helps us to obtain the following:

$$
\begin{aligned}
\left|F_{i}\left(t, u_{1}, u_{2}, \ldots\right)\right|^{2} & \leq 2\left[\frac{(1-t) t}{i^{2} E_{\alpha, B}(t)}\right]^{2}+2 \cdot \frac{E_{\alpha, \beta}^{2}(t)}{(1+i)^{2}} \cdot \frac{\pi^{4}}{90}\left(u_{k}^{2}+\frac{3^{\frac{3}{4}}}{4}\left(\frac{1}{1^{2}}+\frac{1}{2^{2}}+\frac{1}{3^{2}}+\cdots\right)\right) \\
& =2\left[\frac{(1-t) t}{i^{2} E_{\alpha, B}(i t)}\right]^{2}+\frac{3^{\frac{3}{4}} \pi^{4}}{360} \cdot \frac{E_{\alpha, \beta}^{2}(t)}{(1+i)^{2}} \sum_{m=1}^{\infty} \frac{1}{m^{2}}+\frac{\pi^{4}}{45} \cdot \frac{E_{\alpha, \beta}^{2}(t)}{(1+i)^{2}} u_{k}^{2} \\
& =2\left[\frac{(1-t) t}{i^{2} E_{\alpha, B}(i t)}\right]^{2}+\frac{3^{\frac{3}{4}} \pi^{6}}{10,800} \cdot \frac{E_{\alpha, \beta}^{2}(t)}{(1+i)^{2}}+\frac{\pi^{4}}{45} \cdot \frac{E_{\alpha, \beta}^{2}(t)}{(1+i)^{2}} u_{k}^{2} .
\end{aligned}
$$

We now define

$$
\begin{aligned}
& r_{i}=a_{i}+b_{i}:=2\left[\frac{(1-t) t}{i^{2} E_{\alpha, B}(i t)}\right]^{2}+\frac{3^{\frac{3}{4}} \pi^{6}}{10,800} \cdot \frac{E_{\alpha, \beta}^{2}(t)}{(1+i)^{2}}, \\
& q_{i}:=\frac{\pi^{4}}{45} \cdot \frac{E_{\alpha, \beta}^{2}(t)}{(1+i)^{2}} .
\end{aligned}
$$

Here, we need to apply the Weierstrass M-test to identify the validity of the hypotheses (L.2) and (L.3). Since for the real-valued sequence $\left\{r_{i}\right\}_{i=1}^{\infty}$, we have

$$
\begin{aligned}
\left|r_{i}\right|=r_{i} & \leq 2 \frac{\left(\frac{1}{4}\right)^{2}}{i^{4}}+\frac{3^{\frac{3}{4}} \pi^{6}}{10,800} \cdot \frac{E_{\alpha, \beta}^{2}(b)}{(1+i)^{2}} \\
& \leq \underbrace{\left(\frac{1}{8}+\frac{3^{\frac{3}{4}} \pi^{6}}{10,800} E_{\alpha, \beta}^{2}(b)\right) \frac{1}{i^{2}}}_{M_{i}}, \quad i=1,2, \ldots,
\end{aligned}
$$




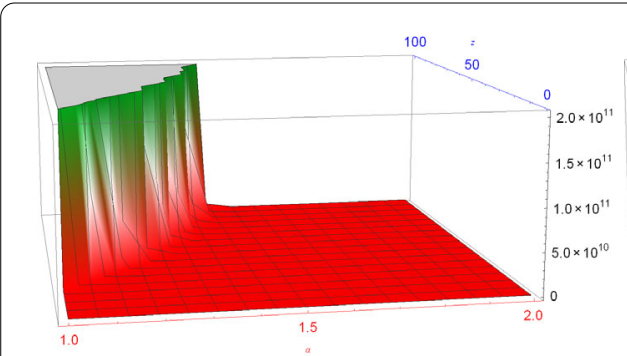

(a) Step 1

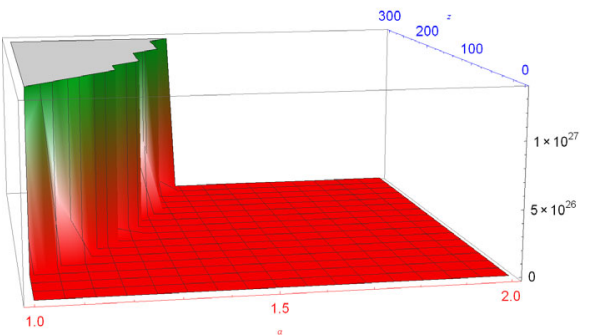

(c) Step 3

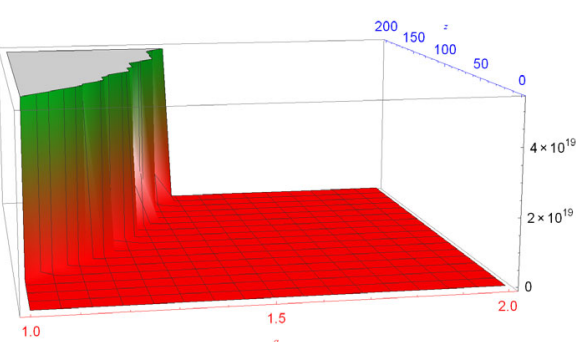

(b) Step 2

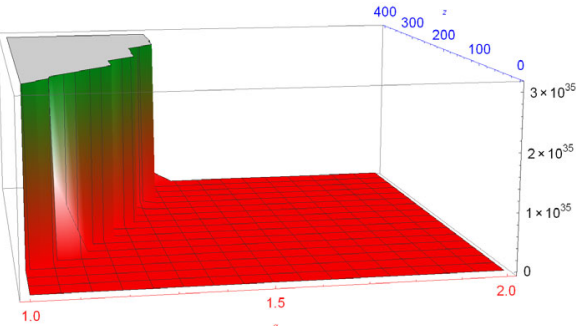

(d) Step 4

Figure 1 Step by step growth of the Mittag-Leffler function $E_{A, B}(z)$ with respect to the growth of $z$

Table 3 Numerical data corresponding to Fig. 1

\begin{tabular}{lll}
\hline$A, B$ & Growth of $z$ & Growth of $E_{A, B}(z)$ \\
\hline$A:=\alpha, B:=A+0.5(2-A)$ & $(a), z \rightarrow 100$ & $E_{A, B}(z) \rightarrow 2 \times 10^{11}$ \\
$A:=\alpha, B:=A+0.5(2-A)$ & $(b), z \rightarrow 200$ & $E_{A, B}(z) \rightarrow 4 \times 10^{19}$ \\
$A:=\alpha, B:=A+0.5(2-A)$ & $(c), z \rightarrow 300$ & $E_{A, B}(z) \rightarrow 1 \times 10^{27}$ \\
$A:=\alpha, B:=A+0.5(2-A)$ & $(d), z \rightarrow 400$ & $E_{A, B}(z) \rightarrow 3 \times 10^{35}$ \\
\hline
\end{tabular}

and since

$$
\sum_{i=1}^{\infty} M_{i}<\infty
$$

it follows from Fig. 1 and Table 3 that the sequence $\sum_{i=1}^{\infty} r_{i}$ is uniformly convergent on $[a, b]$.

Besides, the upper bound estimate

$$
\left|q_{i}\right|=q_{i} \leq \frac{\pi^{6}}{270} E_{\alpha, \beta}^{2}(b)
$$

indicates that the sequence $\left\{q_{i}\right\}_{i=1}^{\infty}$ is equibounded on $[a, b]$. Thus, considering

$$
\left|g_{i}\left(t, u_{1}, u_{2}, \ldots\right)\right|^{2} \leq b_{i}(t)+q_{i}(t) u_{i}^{2}
$$

we arrive at the conclusion that the hypotheses (L.2) and (L.3) are satisfied. Here, we have to prove that the hypothesis (L.1) is also satisfied. To this aim, we consider the operator

$$
(\mathcal{S} u)(t):=\left(F_{1}(t, u), F_{2}(t, u), F_{3}(t, u), \ldots\right)
$$


and fix arbitrary $t \in[a, b]$ and $u=\left(u_{i}\right):=\left(u_{1}, u_{2}, \ldots\right) \in l_{2}$. So, we get

$$
\sum_{i=1}^{\infty}\left|F_{i}\left(t, u_{1}, u_{2}, \ldots\right)\right|^{2} \leq \sum_{i=1}^{\infty} r_{i}(t)+\sum_{i=1}^{\infty} q_{i}(t)\left|u_{i}\right|^{2} \leq \mathcal{A}+\mathcal{Q} \sum_{i=1}^{\infty}\left|u_{i}\right|^{2}
$$

This proves that the operator $\mathcal{S}=\left(F_{i}\right)$ transforms the space $[a, b] \times l_{2}$ into $l_{2}$. Next, we shall take a fixed arbitrary positive constant $\epsilon$ and an arbitrary point $u=\left(u_{i}\right) \in l_{2}$. So, taking the point $v=\left(v_{i}\right) \in l_{2}$ such that $\|u-v\|_{l_{2}}<\epsilon$, it follows that

$$
\begin{aligned}
\|\mathcal{S}(v)-\mathcal{S}(u)\|_{l_{2}} & =\sum_{i=1}^{\infty}\left|F_{i}\left(t, v_{1}, v_{2}, \ldots\right)-F_{i}\left(t, u_{1}, u_{2}, \ldots\right)\right|^{2} \\
& =\sum_{i=1}^{\infty}\left|\sum_{k=i}^{\infty} \frac{E_{\alpha, \beta}(t)}{k^{2}(1+i)} \cdot \frac{v_{k}}{1+(k-i)^{4} v_{k}^{4}}-\sum_{k=i}^{\infty} \frac{E_{\alpha, \beta}(t)}{k^{2}(1+i)} \cdot \frac{u_{k}}{1+(k-i)^{4} u_{k}^{4}}\right|^{2} \\
& =\sum_{i=1}^{\infty}\left|\sum_{k=i}^{\infty} \frac{E_{\alpha, \beta}(t)}{k^{2}(1+i)}\left\{\frac{v_{k}}{1+(k-i)^{4} v_{k}^{4}}-\frac{u_{k}}{1+(k-i)^{4} u_{k}^{4}}\right\}\right|^{2} \\
& \leq \sum_{i=1}^{\infty}\left\{\frac{E_{\alpha, \beta}(t)}{(1+i)} \sum_{k=i}^{\infty} \frac{1}{k^{2}}\left|\frac{v_{k}}{1+(k-i)^{4} v_{k}^{4}}-\frac{u_{k}}{1+(k-i)^{4} u_{k}^{4}}\right|\right\}^{2} \\
& \leq \sum_{i=1}^{\infty} \frac{E_{\alpha, \beta}^{2}(t)}{(1+i)^{2}}\left\{\sum_{k=i}^{\infty} \frac{1}{k^{2}}\left|\frac{v_{k}}{1+(k-i)^{4} v_{k}^{4}}-\frac{u_{k}}{1+(k-i)^{4} u_{k}^{4}}\right|\right\}^{2} .
\end{aligned}
$$

To complete this estimation, we need to apply the Cauchy-Schwarz inequality to obtain the following:

$$
\begin{aligned}
& \|(\mathcal{S} v)(t)-(\mathcal{S} u)(t)\|_{l_{2}}^{2} \\
& \leq \sum_{i=1}^{\infty} \frac{E_{\alpha, \beta}^{2}(t)}{(1+i)^{2}}\left\{\left(\sum_{k=i}^{\infty} \frac{1}{k^{4}}\right)^{\frac{1}{2}}\left(\sum_{k=i}^{\infty}\left|\frac{v_{k}}{1+(k-i)^{4} v_{k}^{4}}-\frac{u_{k}}{1+(k-i)^{4} u_{k}^{4}}\right|^{2}\right)^{\frac{1}{2}}\right\}^{2} \\
& \leq \frac{\pi^{4}}{90} E_{\alpha, \beta}^{2}(t) \sum_{i=1}^{\infty} \frac{1}{(1+i)^{2}}\left\{\sum_{k=i+1}^{\infty}\left[\left|v_{i}(t)-u_{i}(t)\right|^{2}+\left|\frac{v_{k}}{1+(k-i)^{4} v_{k}^{4}}-\frac{u_{k}}{1+(k-i)^{4} u_{k}^{4}}\right|^{2}\right]\right\} \\
& \leq \frac{\pi^{4}}{90} E_{\alpha, \beta}^{2}(t) \sum_{i=1}^{\infty} \frac{1}{(1+i)^{2}} \sum_{k=i}^{\infty}\left|v_{k}(t)-u_{k}(t)\right|^{2} \\
& \leq \frac{\pi^{4}}{90} E_{\alpha, \beta}^{2}(t) \sum_{i=1}^{\infty} \frac{1}{(1+i)^{2}} \epsilon^{2} \\
& \leq \epsilon^{2} \frac{\pi^{6}}{540} E_{\alpha, \beta}^{2}(1) .
\end{aligned}
$$

If we summarize the above process, we conclude that

$$
\|(\mathcal{S} v)(t)-(\mathcal{S} u)(t)\|_{l_{2}} \leq \epsilon \frac{\pi^{3}}{6 \sqrt{15}} E_{\alpha, \beta}^{2}(1), \quad t \in[0,1]
$$


So, the recent inequality ensures that the hypothesis (L.1) is satisfied. Finally, choosing $\alpha:=1.5, \beta:=0.5$ and $\pi \approx 3.1416$, we get

$$
\Lambda^{p}(b-a)^{\frac{p+q}{q}} Q \approx 0.961703<1 .
$$

Therefore, since all conditions of Theorem 3.2 hold, it has been proved that the infinite system of the Hilfer fractional boundary value problems (4.1) has at least one solution $u(t)=\left(u_{i}(t)\right)$ in the sequence space $C\left([a, b] ; l_{2}\right)$.

Example 4.2 In order to illustrate the applicability of the obtained theoretical existence criterion in Theorem 3.1, we consider the following infinite system of the Hilfer fractional boundary value problems:

$$
\mathcal{D}_{a^{+}}^{\alpha, \beta} u_{i}=-a_{i}(t)-g_{i}\left(t, u_{1}, u_{2}, \ldots\right), \quad u_{i}(a)=u_{i}(b)=0, a<t<b, i=1,2, \ldots
$$

under the following setting:

$$
\begin{aligned}
& a_{i}(t)=:=\frac{\sqrt{t} E_{A, B}(-i t)}{1+i^{2}} \\
& g_{i}\left(t, u_{1}, u_{2}, \ldots\right):=\sum_{k=i}^{\infty} \frac{\cos (t)}{(1+i)^{4}} \cdot \frac{u_{k}}{1+k^{4} u_{k}^{2}}, \quad i=1,2, \ldots
\end{aligned}
$$

We assume that all of the parameters in this application are the same as those in Example 4.1. To see that the infinite system (4.9) has at least one solution in the sequence space $c_{0}$, we have to show that all conditions of Theorem 3.1 hold. To this aim, first one can observe that

$$
\max _{\omega>0} \frac{\omega}{1+\rho^{4} \omega^{2}}:=\frac{1}{2 \rho^{2}}, \quad \rho>0 .
$$

So, if $u(t)=\left(u_{i}(t)\right) \in c_{0}$, then, by the use of this fact, we get the following:

$$
\begin{aligned}
\lim _{i \rightarrow \infty} F_{i}(t, u) & =\lim _{i \rightarrow \infty}\left(\frac{\sqrt{t} E_{A, B}(-i t)}{1+i^{2}}+\sum_{k=i}^{\infty} \frac{\cos (t)}{(1+i)^{4}} \cdot \frac{u_{k}}{1+k^{4} u_{k}^{2}}\right) \\
& \leq \lim _{i \rightarrow \infty}\left(\frac{\sqrt{t} E_{A, B}(-i t)}{1+i^{2}}+\frac{\cos (t)}{(1+i)^{4}} \cdot \sum_{k=0}^{\infty} \frac{1}{2 k^{2}}\right) \\
& =\lim _{i \rightarrow \infty}\left(\frac{\sqrt{t} E_{A, B}(-i t)}{1+i^{2}}+\frac{\pi^{2} \cos (t)}{12(1+i)^{4}}\right)=0,
\end{aligned}
$$

that is, $F_{i}(t, u) \in c_{0}$. Since the proof of equicontinuity of the operator $\mathcal{Q}:[a, b] \times c_{0} \rightarrow c_{0}$,

$$
(\mathcal{Q} u)(t):=\left(F_{1}(t, u), F_{2}(t, u), \ldots\right)
$$

is similar to that of in Example 4.1, so we omit it here. Hence, we begin with the hypothesis (C.2) as follows. According to (4.10), it is easy to check that $a_{i}(t) \in C\left([a, b] ; \mathbb{R}^{+}\right)$for $i=$ $1,2, \ldots$ Also, based on Fig. 2 and its corresponding numerical data Table 4 , as represented 


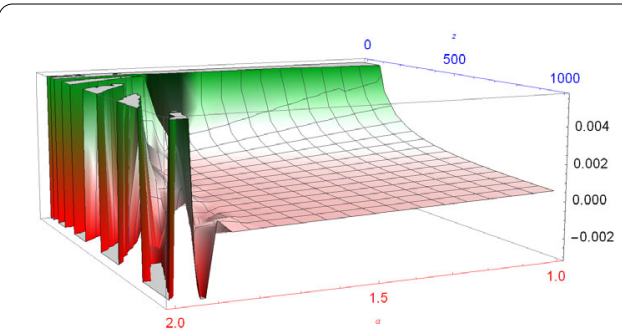

(a) Step 1

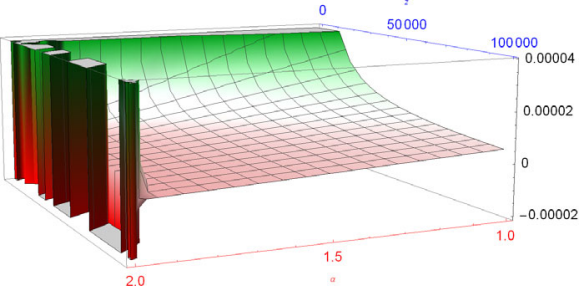

(c) Step 3

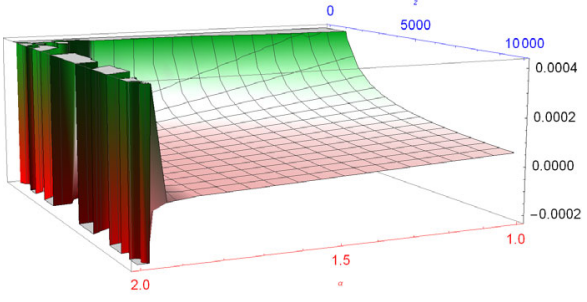

(b) Step 2

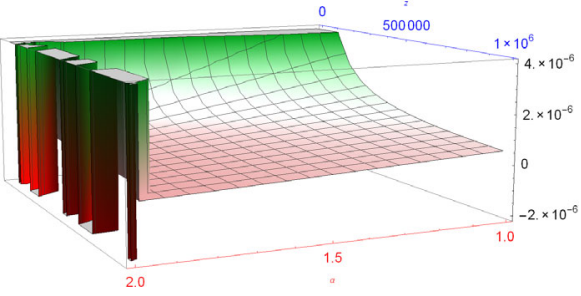

(d) Step 4

Figure 2 Step by step decrement of the Mittag-Leffler function $E_{A, B}(-z)$ with respect to the growth of $z$

Table 4 Numerical data corresponding to Fig. 2

\begin{tabular}{lll}
\hline$A, B$ & Growth of $z$ & Decrement of $E_{A, B}(-z)$ \\
\hline$A:=\alpha, B:=A+0.5(2-A)$ & $(a), z \rightarrow 10^{3}$ & $E_{A, B}(-z) \rightarrow 4 \times 10^{-3}$ \\
$A:=\alpha, B:=A+0.5(2-A)$ & $(b), z \rightarrow 10^{4}$ & $E_{A, B}(-z) \rightarrow 4 \times 10^{-4}$ \\
$A:=\alpha, B:=A+0.5(2-A)$ & $(c), z \rightarrow 10^{5}$ & $E_{A, B}(-z) \rightarrow 4 \times 10^{-5}$ \\
$A:=\alpha, B:=A+0.5(2-A)$ & $(d), z \rightarrow 10^{6}$ & $E_{A, B}(-z) \rightarrow 4 \times 10^{-6}$ \\
\hline
\end{tabular}

below, we arrive at the conclusion that $\left\{a_{i}\right\}_{i=1}^{\infty}$ vanishes identically on $[a, b]$, that is, the hypothesis (C.2) is satisfied.

Here we consider the functions $g_{i}\left(t, u_{1}, u_{2}, \ldots\right), i=1,2, \ldots$ Thus, we have

$$
\begin{aligned}
\left|g_{i}\left(t, u_{1}, u_{2}, \ldots\right)\right| & =\left|\sum_{k=i}^{\infty} \frac{\cos (t)}{(1+i)^{4}} \cdot \frac{u_{k}}{1+k^{4} u_{k}^{2}}\right| \\
& \leq \frac{\cos (t)}{(1+i)^{4}} \cdot\left|\sum_{k=i}^{\infty} \frac{u_{k}}{1+k^{4} u_{k}^{2}}\right| \\
& \leq \frac{\cos (t)}{(1+i)^{4}}\left(\left|u_{k}\right|+\frac{1}{2}\left\{\frac{1}{1 !}+\frac{1}{2 !}+\frac{1}{3 !}+\cdots\right\}\right) \\
& \leq \frac{\pi^{2} \cos (t)}{12(1+i)^{4}}+\frac{\cos (t)}{(1+i)^{4}} \sup _{k \geq i}\left\{\left|u_{k}\right|\right\} \\
& :=b_{i}(t)+q_{i}(t) \sup _{k \geq i}\left\{\left|u_{k}\right|\right\} .
\end{aligned}
$$

Now, it is easy to check that the hypothesis (C.3) is also satisfied. Finally, we have to check that the assumption $\Lambda(b-a) \mathcal{Q}$ holds. Since $\Lambda \approx 0.434314, a:=0, b:=1$ and $\mathcal{Q}:=1$, it 
follows that

$$
\Lambda(b-a) \mathcal{Q} \approx 0.434314<1 \text {. }
$$

Therefore, Theorem 3.2 ensures that the infinite system of Hilfer fractional boundary value problems (4.9) has at least one solution $u(t):=\left(u_{i}(t)\right)$ in $c_{0}$.

\section{Acknowledgements}

The author gratefully acknowledges the editor in charge and all of the anonymous three referees for detailed and constructive suggestions.

\section{Funding}

Not applicable.

Availability of data and materials

Data sharing not applicable to this paper as no datasets were generated or analyzed during the current study.

\section{Competing interests}

The author declares that he has no competing interests.

\section{Authors' contributions}

The author read and approved the final version of the current paper.

\section{Publisher's Note}

Springer Nature remains neutral with regard to jurisdictional claims in published maps and institutional affiliations.

Received: 17 August 2020 Accepted: 21 February 2021 Published online: 04 March 2021

\section{References}

1. Abbas, S., Agarwal, R.P., Benchohra, M., Lazreg, J., Ahmad, B.: Bounded weak solutions for Hilfer fractional differential equations on the half line. Adv. Dyn. Syst. Appl. 15(1), 35-49 (2020)

2. Aghajani, A., Allahyari, R., Mursaleen, M.: A generalization of Darbo's theorem with application to the solvability of systems of integral equations. J. Comput. Appl. Math. 260, 68-77 (2014)

3. Aghajani, A., Mursaleen, M., Haghighi, A.S.: Fixed point theorems for Meir-Keeler condensing operators via measure of noncompactness. Acta Math. Sci. Ser. B Engl. Ed. 35(3), 552-566 (2015)

4. Akmerov, R.R., Kamenski, M.I., Potapov, A.S., Rodkina, A.E., Sadovskii, B.N.: Measures of Noncompactness and Condensing Operators. Birkhauser, Basel (1992)

5. Atanackovic, T.M., Pilipovic, S., Stankovic, B., Zorica, D.: Fractional Calculus with Applications in Mechanics. Wiley, New York (2014)

6. Ayerbe Toledano, J.M., Benavides, T.D., Acedo, G.L.: Measures of Noncompactness in Metric Fixed Point Theory. Birkhäuser, Basel (1997)

7. Baleanu, D., Guvenc, Z.B., Machado, J.A.T.: New Trends in Nanotechnology and Fractional Calculus Applications. Springer, Berlin (2010)

8. Baleanu, D., Machado, J.A.T., Luo, A.C.J.: Fractional Dynamics and Control. Springer, Berlin (2012)

9. Banas, J., Goebel, K.: Measures of Noncompactness in Banach Spaces. Lecture Notes in Pure and Applied Mathematics, vol. 60. Dekker, New York (1980)

10. Banas, J., Mursallen, M.: Sequence Spaces and Measures of Noncompactness with Applications to Differential and Integral Equations. Springer, Berlin (2014)

11. Bedi, P., Kumar, A., Abdeljawad, T., Khan, A.: Existence of mild solutions for impulsive neutral Hilfer fractional evolution equations. Adv. Differ. Equ. 2020, 155 (2020). https://doi.org/10.1186/s13662-020-02615-y

12. Bhairat, S.P.: Existence and continuation of solutions of Hilfer fractional differential equations. J. Math. Model. 7(1), $1-20(2019)$

13. Gao, W., Veeresha, P., Baskonus, H.M., Prakasha, D.G., Kumar, P.: A new study of unreported cases of 2019-nCOV epidemic outbreaks. Chaos Solitons Fractals 138, 109929 (2020)

14. Gu, H., Trujillo, J.J.: Existence of mild solution for evolution equation with Hilfer fractional derivative. Appl. Math Comput. 257, 344-354 (2015)

15. Hilfer, R.: Applications of Fractional Calculus in Physics. World Scientific, Singapore (2000)

16. Kilbas, A.A., Srivastava, H.M., Trujillo, J.J.: Theory and Applications of Fractional Differential Equations. North-Holland Mathematics Studies, Elsevier Science, vol. 204 (2006)

17. Kuratowski, K.: Sur les espaces complets. Fundam. Math. 5, 301-309 (1930)

18. Lewis, D.C. Jr: Infinite systems of ordinary differential equations with applications to certain second-order partial differential equations. Trans. Am. Math. Soc. 35(4), 792-823 (1933)

19. Liu, X., Li, Y., Xu, G.: On the finite approximate controllability for Hilfer fractional evolution systems. Adv. Differ. Equ. 2020, 22 (2020). https://doi.org/10.1186/s13662-019-2478-5

20. Liu, J., Yang, X., Cheng, M., Feng, Y., Wang, Y.: Abound rogue wave type solutions to the extended $(3+1)$-dimensional Jimbo-Miwa equation. Comput. Math. Appl. 78(6), 1947-1959 (2019) 
21. Mainardi, F.: Fractional Calculus and Waves in Linear Viscoelasticity. World Scientific, Singapore (2010)

22. Miller, K.S., Ross, B.: An Introduction to Fractional Calculus and Fractional Differential Equation. Wiley, New York (1993)

23. Mursaleen, M., Rizvi, S.M.H.: Solvability of infinite systems of second order differential equations in $c_{0}$ and $l_{1}$ by Meir-Keeler condensing operators. Proc. Am. Math. Soc. 144, 4279-4289 (2016)

24. Mursaleen, M., Mohiuddine, S.A.: Applications of measures of noncompactness to the infinite system of differential equations in Is spaces. Nonlinear Anal. TMA 75, 2111-2115 (2012)

25. Oldham, K.B., Spanier, J.: The Fractional Calculus. Academic Press, New York (1974)

26. Pathak, N.: Lyapunov-type inequality for fractional boundary value problems with Hilfer fractional derivative. Math, Inequal. Appl. 21(1), 179-200 (2018)

27. Petras, I.: Fractional-Order Nonlinear Systems. Modeling, Analysis and Simulation. Springer, Berlin (2011)

28. Podlubny, I.: Fractional Differential Equations. In: Mathematics in Science and Applications, p. 19. Academic Press, New York (1999)

29. Ray, S.S.: Fractional Calculus with Applications for Nuclear Reactor Dynamics. CRC Press, Boca Raton (2016)

30. Steinberg, S.: Infinite systems of ordinary differential equations with unbounded coefficients and moment problems. J. Math. Anal. Appl. 41, 685-694 (1973)

31. Subashini, R., Ravichandran, C.: On the results of nonlocal Hilfer fractional semilinear differential inclusions. Proc. Jangjeon Math. Soc. 22(2), 249-267 (2019)

32. Subashini, R., Ravichandran, C., Jothimani, K., Baskonus, H.M.: Existence results of Hilfer integro-differential equations with fractional order. Discrete Contin. Dyn. Syst., Ser. S 13(3), 911-923 (2020)

33. Tomovski, Z: Generalized Cauchy type problems for nonlinear fractional differential equations with composite fractional derivative operator. Nonlinear Anal. TMA 75, 3364-3384 (2012)

34. Valliammal, N., Ravichandran, C., Hammouch, Z., Baskonus, H.M.: A new investigation on fractional-ordered neutral differential systems with state-dependent delay. Int. J. Nonlinear Sci. Numer. Simul. 20(7-8), 803-809 (2019)

35. Voigt, A.: Line method approximations to the Cauchy problem for nonlinear parabolic differential equations. Numer. Math. 23, 23-36 (1974)

36. Yang, X., Gao, F., Ju, Y., Zhou, H.: Fundamental solutions of the general fractional-order diffusion equations. Math. Methods Appl. Sci. 41, 9312-9320 (2018)

37. Yang, X.: General Fractional Derivatives: Theory, Methods and Applications. CRC Press, New York (2019)

38. Yang, X., Gao, F., Ju, Y.: General Fractional Derivatives with Applications in Viscoelasticity. Academic Press, San Diego (2020)

39. Yang, X., Machado, J.A.T.: A new fractal nonlinear Burgers' equation arising in the acoustic signals propagation. Math. Methods Appl. Sci. 42(18), 7539-7544 (2019)

40. Yang, X., Abel-Aty, M., Cattani, C.: A new general fractional-order derivative with Rabotnov fractional-exponential kernel applied to model the anomalous heat transfer. Therm. Sci. 23(3A), 1677-1681 (2019)

\section{Submit your manuscript to a SpringerOpen ${ }^{\circ}$ journal and benefit from:}

- Convenient online submission

- Rigorous peer review

- Open access: articles freely available online

- High visibility within the field

- Retaining the copyright to your article

Submit your next manuscript at $\gg$ springeropen.com 